\title{
A microdeletion in 19q13.2 associated with mental retardation, skeletal malformations, and Diamond-Blackfan anaemia suggests a novel contiguous gene syndrome
}

\author{
Dmitry Tentler, Peter Gustavsson, Göran Elinder, Ole Eklöf, Laurie Gordon, \\ Ariane Mandel, Niklas Dahl
}

\begin{abstract}
Diamond-Blackfan anaemia (DBA) is a constitutional red blood cell hypoplasia which may be associated with a variety of developmental abnormalities. A gene for DBA was recently mapped to chromosome 19q13.2 and subsequently cloned. Analysis of 19q marker alleles in DNA of sporadic DBA cases showed de novo microdeletions in three patients also presenting with mental retardation. We have studied one of these patients and characterised the deletion by fluorescence in situ hybridisation (FISH) to extended DNA fibres. The deletion was shown to be continuous over a $3.2 \mathrm{Mb}$ region and the fibre-FISH analysis showed both chromosomal breakpoints. In combination, the clinical and molecular findings suggest a contiguous gene syndrome with a gene locus for mental retardation and, probably, skeletal malformations included in the deletion.

(F Med Genet 2000;37:128-131)
\end{abstract}

Unit of Clinical Genetics, Department of Genetics and Pathology, Uppsala University Children's Hospital, S-751 85 Uppsala, Sweden D Tentler

P Gustavsson

A Mandel

N Dahl

Department of Paediatrics, Institution of Södersjukhuset, Karolinska Institute, Stockholm, Sweden G Elinder

Department of Paediatric Radiology, Karolinska Hospital, Stockholm, Sweden O Eklöf

Lawrence Livermore National Laboratory, Livermore, CA, USA L Gordon

Correspondence to: Dr Dahl

Revised version received 13 September 1999 Accepted for publication 23 September 1999
Keywords: chromosome 19q13; microdeletion syndrome; fibre-FISH

Diamond-Blackfan anaemia (DBA, McKusick 205900) is a red cell hypoplasia characterised by subnormal erythropoiesis with absent or decreased red cell precursors in the bone marrow. ${ }^{12}$ A subset of patients has associated phenotypic abnormalities including thumb malformations, urogenital abnormalities, atrial or ventricular septal defects, prenatal or postnatal growth retardation, learning difficulties, strabismus, and cataract. ${ }^{3-5}$

At least $10 \%$ of patients with DBA have a positive family history for the disorder following either autosomal dominant or autosomal recessive inheritance. One locus for DBA has been mapped to chromosome 19q13.2 in multiplex families ${ }^{6}$ and we recently cloned the gene which was shown to encode the ribosomal protein (RP) S19. ${ }^{7}$ We have analysed 19q13 marker alleles which flank the RPS19 gene in 50 sporadic cases with DBA. Three subjects also with mental retardation (MR) were found to be hemizygous for several marker loci. ${ }^{8}$ One of these patients with microdeletions was recently reported ${ }^{9}$ and we present here the second of the three patients. The clinical features of this patient are described as well as the pre- cise mapping of the deletion breakpoints using FISH to extended DNA fibres. The complex phenotype including mental retardation and generalised skeletal malformations suggests a contiguous gene syndrome.

\section{Case report}

The patient is a 12 year old male, born at term after an uncomplicated pregnancy to healthy, unrelated parents. There is no family history of skeletal, mental, or haematological disorders. At the age of 2 months he was referred to a paediatric clinic because of pallor. Haemoglobin concentration was $4.8 \mathrm{~g} / \mathrm{dl}$ and no reticulocytes were detected in peripheral blood. Bone marrow aspirate showed a selective decrease in erythroid precursors but otherwise normal cellularity. Chromosome analysis (G banding) showed a normal 46,XY karyotype and chromosome fragility test with mitomycin $\mathrm{C}$ was normal. At 22 months of age the boy had relatively short extremities, slight macrocephaly (+2 SD), and short stature ( -3 $\mathrm{SD})$. Major radiological traits recorded at 1 year 10 months of age included 13 pairs of horizontally oriented ribs, mild thoracic platyspondyly, vertebral bone in bone formation, and lack of interpedicular widening with ventrally concave posterior aspects of the lumbar vertebrae (fig 1A). The iliac bodies were narrow, the proximal femoral epiphyses asymmetrical, and the smaller right one in addition slightly irregular (fig 1B). Coxa valga with short and broad femoral necks as well as bow legs with epiphyseal flare were also noted (fig 1C). Radiological investigations of the hands were normal.

Moderate psychomotor retardation was diagnosed at the age of 4 years. A cranial CT scan at this age was normal, as were ultrasound and $x$ ray investigations of the heart and lungs. MRI of the liver and spleen at 7 years of age showed iron accumulation.

At 12 years of age he requires transfusions every three weeks. His height is $140 \mathrm{~cm}(-1.5$ $\mathrm{SD})$, weight is $36 \mathrm{~kg}(-0.5 \mathrm{SD})$, and head circumference is $58.2 \mathrm{~cm}(+2 \mathrm{SD})$. The span of the upper extremities is $131 \mathrm{~cm}$ and his sitting height is $73 \mathrm{~cm}$. No follow up $x$ ray has been performed.

\section{Materials and methods}

FIBRE-FISH ANALYSIS

In order to determine the deletion breakpoints and for a precise size estimation of the deletion 

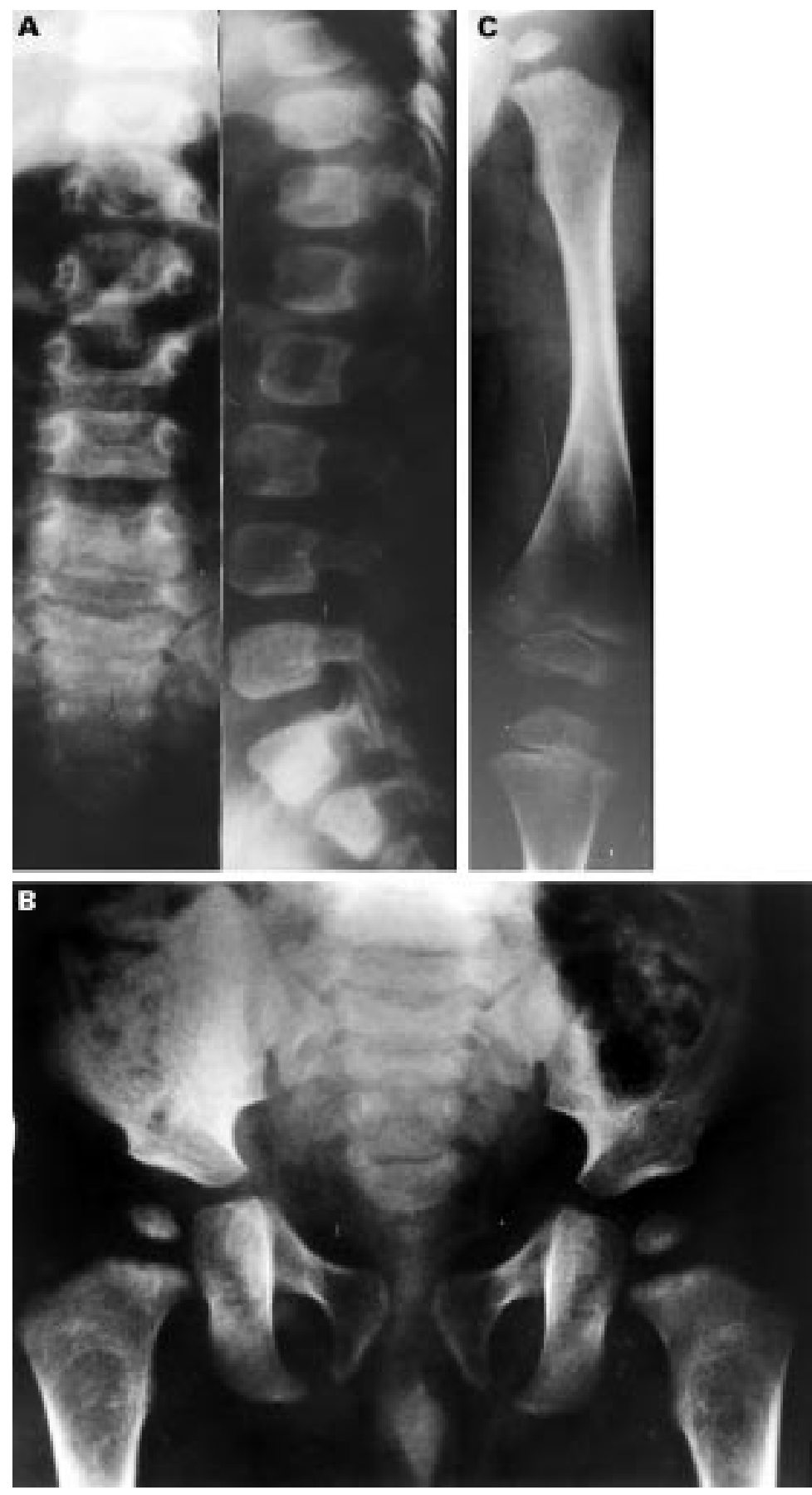

Figure 1 Radiological survey of patient $R G$ at the age of 1 year 10 months. (A) Anterior-posterior and lateral films of the spine show slight thoracic platyspondyly, bone in bone formation, lack of interpedicular widening, and anteriorly concave posterior aspects of the lumbar vertebrae. (B) Pelvic $x$ ray shows narrow iliac bodies, asymmetrical proximal femoral epiphyses, the smaller right one of which is irregular. Coxa valga with short, broad femoral necks can be seen. (C) Left lower femur and knee show a relative mid diaphyseal constriction and $a V$ shaped flare of the distal femoral metaphyses. Metaphyseal sclerosis and irregularity can be seen. The epiphyses are small and flattened, with somewhat irregular epiphyses.

we used fibre-FISH analysis. Fluorescence in situ hybridisation to extended DNA fibres was performed essentially as described elsewhere. ${ }^{10}{ }^{11}$ Unfixed linearised DNA fibres were prepared on microscope slides. Ficoll separated lymphoblastoid cells derived from the patient and lymphoblastoid cells from a control were embedded in $1 \%$ low melting point agarose at a concentration of $10^{7}$ cells $/ \mathrm{ml}$.
Agarose blocks were incubated at $50^{\circ} \mathrm{C}$ in 2 $\mathrm{mg} / \mathrm{ml}$ proteinase $\mathrm{K}$ and $1 \%$ $\mathrm{N}$-lauroylsarcosine in $50 \mathrm{mmol} / 1 \mathrm{EDTA}$ for 48 hours. A small piece of agarose embedded DNA was placed on a microscope slide and 15 $\mu l$ of water was added to the agarose block. The slide was heated on a heat block (50 seconds, $100^{\circ} \mathrm{C}$ ) and DNA was extended manually on a slide using the edge of another microscope slide. Extended DNA fibres were denatured using $70 \%$ formamide $/ 2 \times$ SSC at $74^{\circ} \mathrm{C}$ for four minutes followed by an ice cold ethanol series. Cosmid DNA was labelled either with biotin or digoxigenin using nick translation. Double hybridisation was detected using a single layer of FITC-avidin (Vector Laboratories) and rhodamine labelled anti-digoxigenin antibodies (Boehringer Mannheim). Visualisation of probes was performed as described previously. ${ }^{6} 1112$

\section{COSMID CLONES}

The probe and interprobe distances in kilobases were based on a detailed physical map of the region. ${ }^{13}$ The chromosome 19 cosmids were obtained from the Lawrence Livermore National Laboratory (CA) with their corresponding numbers. In total, nine cosmids were used for fibre-FISH analysis and their relative order from the centromere to the telomere is $15848,16767,22692,16923,8918,15894$, 26656, 28280, and 29827.

\section{Results}

Cosmid clones assigned to the chromosome 19 q13 map identified the deletion by FISH to metaphase chromosomes from patient RG. The extremities of the deletion were approximately mapped between, and close to, cosmids 15848 (CYP2B6) on the centromeric side and 13519 (D19S19) on the telomeric side. ${ }^{6}$ Additional cosmids in the vicinity of loci CYP2B6 and D19S19 were subsequently hybridised to DNA fibres derived from patient RG and from a normal control, respectively. Two to three cosmids of known relative position and size (38-45 kb) were cohybridised and the clones were distinguished by two colour detection.

Hybridisation with cosmid 22692 to DNA fibres derived from the patient showed two different signal patterns corresponding to a length of approximately $40 \mathrm{~kb}$ and $15 \mathrm{~kb}$, respectively. Similarly, hybridisation with cosmid 28280 showed two types of signals corresponding to either $40 \mathrm{~kb}$ or $20 \mathrm{~kb}$. We then cohybridised the two rearranged cosmids 22692 and 28280 together with the control cosmid 29827 to extended DNA fibres from the patient. The results showed that signals of cosmids 22692 and 28280 , normally separated by $3.2 \mathrm{Mb}$, were located head to tail on approximately half the numbers of fibres (fig 2B). In the same experiment, separate and normal signals from cosmids 28280 and 22692 were also detected (fig 2A). These signals are expected from the non-rearranged fibres. The combined results indicate that the deletion is continuous and that the breakpoints are located within cosmid 22692 (centromeric) and within cosmid 28280 (telomeric). 

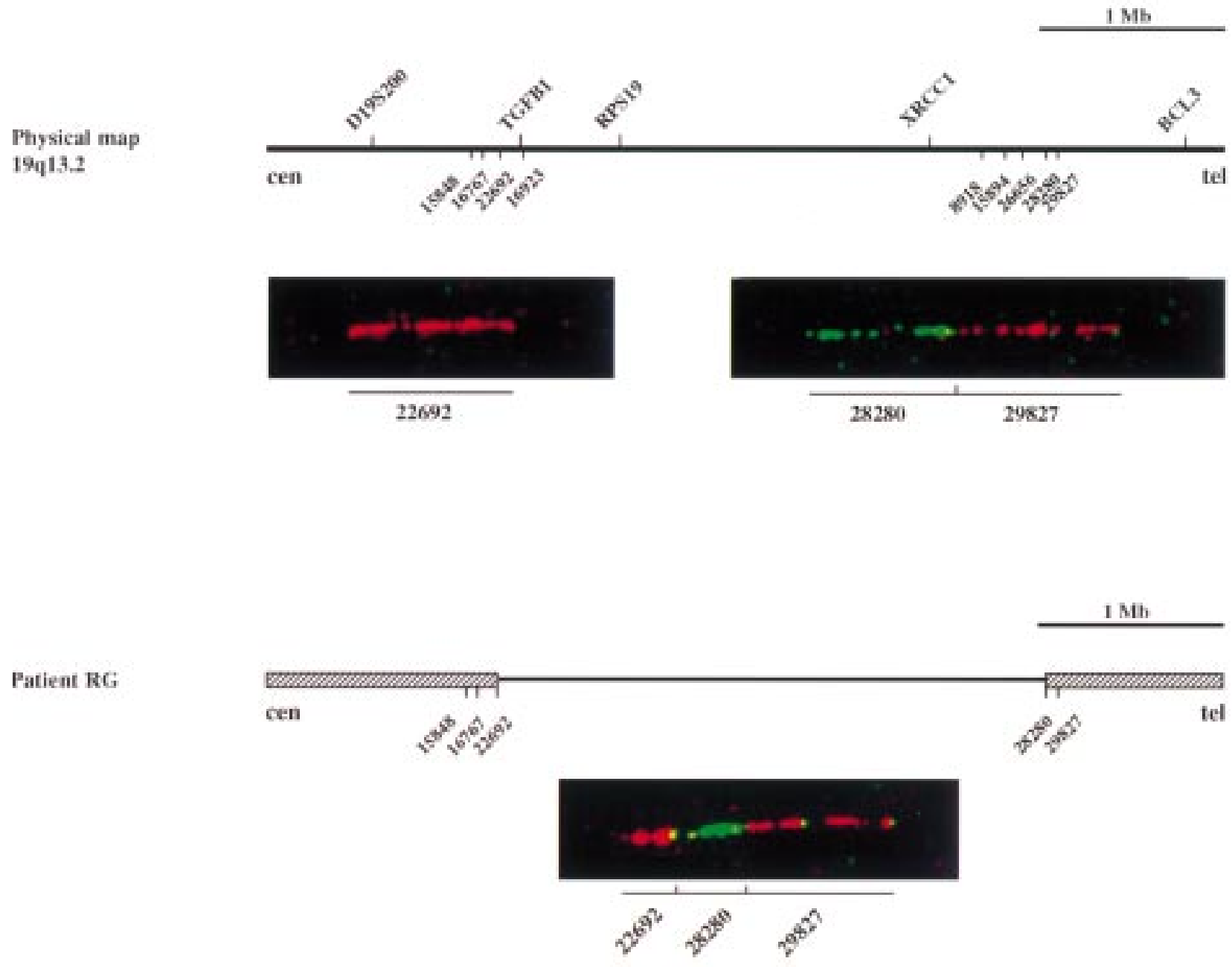

Figure 2 Fibre-FISH mapping of the 19q13.2 region in patient RG. (Above) Physical map of part of the $19 q 13$ region corresponding to the deletion (top). The normal alleles are visualised on extended fibres after cohybridisation with three cosmids (below). Cosmid 22692 (red) is located $3.2 \mathrm{Mb}$ centromeric to the tandemly arrayed cosmid 28280 (green) and the control cosmid 29827 (red). (Below) Schematic map of the deletion in patient RG (top). The striped bars denote part of the chromosome that is present. The hybridisation pattern from a rearranged fibre is shown below. Signals from cosmids 22692 and 28280 are shortened and positioned head to tail. The signal corresponding to cosmid 29827 appears normal.

\section{Discussion}

We have characterised a $3.2 \mathrm{Mb}$ microdeletion in chromosome $19 \mathrm{q} 13.2$ associated with DBA, mental retardation, and skeletal abnormalities. Fibre-FISH analysis enabled the deletion breakpoints in patient RG to be visualised at a high resolution and the deletion was found to be continuous. The deleted region spans more than 20 known genes ${ }^{1314}$ and there are additional expressed sequence tags (ESTs) mapped to $19 \mathrm{q} 13.2$. Several gene families are clustered in a region corresponding to the deletion including the $C Y P 2$, the $C G M$, and the $P S G$ gene families. It is known that tandemly repeated and homologous sequences may predispose to recombination events. ${ }^{15} 16$ However, such a mutation mechanism needs to be verified by DNA sequencing of the deletion breakpoints in our patient.

The combined clinical and molecular findings suggest that the patient has a contiguous gene syndrome. The gene encoding RPS19, previously shown to be mutated in a subset of DBA patients, is included in the deletion. The involvement of other genes in the patient's phenotype is substantiated by the recent clinical presentation of a boy with DBA, psychomotor retardation, and dysplastic changes of bone metaphyses and the vertebral column. ${ }^{9}$ This patient carries a deletion of approximately 3 $\mathrm{Mb}$ which, although not precisely characterised, overlaps with the one found in our patient. Additional support for a contiguous gene syndrome comes from the investigation of a female with DBA and an associated balanced X;19 translocation which interrupts the RPS19 gene. ${ }^{72}$ This female is haploinsufficient for the $D B A$ gene on $19 \mathrm{q} 13.2$ and she has normal mental development with no skeletal malformations. In the subset of DBA patients screened for mutations in the RPS19 gene, ${ }^{7}$ none of the cases caused by point mutations in RPS19 had psychomotor retardation, while patients with abnormal mental development were found to carry deletions in the $19 \mathrm{q} 13.2$ region. ${ }^{8}$ The data point to the presence of a gene responsible for mental retardation linked to the RPS19 gene. 
Our patient presents with some physical abnormalities, for example, short stature, that have been described previously as frequently associated with DBA. ${ }^{1245}$ However, there are very few published cases of DBA patients with malformations of the lower limbs, pelvis, and vertebrae. $^{916}$ The similar phenotype in our patient may indicate a gene for the skeletal malformations involved in the deletion.

In conclusion, the presence of complex clinical features in our patient suggests the involvement of a gene for mental retardation and, probably, skeletal abnormalities linked to the RPS19 gene. The finding of such uncommon features in a small number of DBA patients may be explained by deletions affecting contiguous genes. The comparison of characterised deletions associated with DBA may further delineate the genomic regions responsible for the complex phenotype of this patient.

We thank Dr Aarno Palotie for advice. We also thank the DBA working groups and the ESPHI. This study was supported by grants from the Children's Cancer Foundation of Sweden, the Swedish Medical Research Council, DBA Foundation Inc, the Sävstaholm Society, Harald Jeansson's Foundation, Torsten och Ragnar Söderberg's Foundation, Selander's Foundation, Lundberg Foundation, and R MacDonald's fund. Work at the LLNL was performed under the auspices of the US Department of Energy under Contract W-7405-ENG-48.

1 Halperin DS, Freedman MH. Diamond-Blackfan anemia: etiology, pathophysiology, and treatment. Am f Pediatr Hematol Oncol 1989;11:380-94.

2 Young NS, Alter BP, eds. Aplastic anemia: acquired and inherited. Philadelphia: Saunders, 1994.

3 Aase JM, Smith DW. Congenital anemia and triphalangeal thumbs: a new syndrome. Pediatrics 1969;74:471-4.
4 Ball SE, McGuckin CP, Jenkins G, Gordon-Smith EC. Diamond-Blackfan anemia in the UK: analysis of 80 cases from a 20-year birth cohort. Br F Haematol 1996;94:64553

5 Janov AJ, Leong T, Nathan DG, Guinan EC. Diamondment. Medicine 1996;75:77-87.

6 Gustavsson P, Willig TN, van Haeringen A, et al. DiamondBlackfan anaemia: genetic homogeneity for a gene on chromosome $19 \mathrm{q} 13$ restricted to $1.8 \mathrm{Mb}$. Nat Genet 1997;16: 368-71.

7 Draptchinskaia N, Gustavsson P, Andersson B, et al. The gene encoding ribosomal protein $\mathrm{S} 19$ is mutated in Diamond-Blackfan anaemia. Nat Genet 1999;21:169-75.

8 Gustavsson P, Garelli E, Draptchinskaia N, et al. Identification of microdeletions spanning the Diamond-Blackfan anemia (DBA) locus on 19q13 and evidence for genetic heterogeneity. Am f Hum Genet 1998;63:1388-95.

9 Cario H, Gustavsson P, Dahl N, Kohne E. A microdeletion syndrome due to a 3-Mb deletion on 19q13.2-DiamondBlackfan anemia associated with macrocephaly, hypotonia, and psychomotor retardation. Clin Genet 1999;55:487-92.

10 Heiskanen M, Hellsten E, Kallioniemi OP, et al. Visual mapping by fiber-FISH. Genomics 1995;30:31-6.

11 Tentler D, Gustavsson P, Leisti J, et al. Deletion including the oligophrenin-1 gene associated with enlarged cerebral ventricles, cerebellar hypoplasia, seizures, ataxia and androgen insensitivity. Eur F Hum Genet 1999;7:541-8.

12 Gustavsson P, Skeppner G, Johansson B, et al. DiamondBlackfan anaemia in a girl with a de novo balanced reciprocal X;19 translocation. F Med Genet 1997;34:779-82.

13 Ashworth LK, Batzer MA, Brandriff B, et al. An integrated metric physical map of human chromosome 19. Nat Genet 1995;11:422-7.

14 Mohrenweiser H, Olsen A, Archibald A, et al. Report of the third international workshop on human chromosome 19 mapping 1996. Cytogenet Cell Genet 1996;74:161-86.

15 Pascoe L, Curnow KM, Slutsker L, et al. Glucocortoidsuppressible hyperaldosteronism results from hybrid genes created by unequal crossovers between CYP11B1 an CYP11B2. Proc Natl Acad Sci USA 1992;89:8327-31.

16 Frangiskakis JM, Ewart AK, Morris CA, et al. LIM-kinase1 hemizygosity implicated in impaired visuospatial constructive cognition. Cell 1996;86:59-69.

17 Hing AV, Dowton SB. Aase syndrome: novel radiographic features. Am ₹ Med Genet 1993;45:413-15. 